\title{
Analyse sociospatiale du clivage sexué des pratiques du vélo durant l'adolescence
}

\author{
Sociospatial analysis of gendered cycling practices during adolescence
}

\author{
David Sayagh \\ (C) IFSTTAR et Éditions NecPlus 2016
}

Résumé Les adolescents sont beaucoup plus nombreux que les adolescentes à déclarer faire du vélo. L'examen combiné de travaux liés à l'univers des activités physiques et à celui des pratiques de mobilité, conduit à se demander si certains déterminants impactent différemment les filles et les garçons. Les estimations résultant d'un modèle de régression logistique polytomique ordinal élaboré à partir de l'ENTD (Enquête nationale transports déplacements) 2008, révèlent que l'effet du sexe ne diffère significativement ni selon la « catégorie d'unité urbaine », ni selon le « diplôme le plus élevé de la PR », ni selon le « revenu simulé par UC ». En outre, nous montrons d'une part que l'écart entre garçons et filles vis-à-vis de la probabilité de faire du vélo n'est jamais aussi creusé qu'en ZUS (zone urbaine sensible), et d'autre part, que si au cours de l'adolescence, cette probabilité décroît pour les garçons comme pour les filles, l'écroulement s'avère plus prononcé et essentiellement concentré entre la «préadolescence » et la « fin des années collège » chez les filles.

Mots clés vélo $\cdot$ adolescence $\cdot$ activité physique $\cdot$ pratique sexuée · ZUS

\begin{abstract}
Teenage boys are much more likely to bicycle than teenage girls. Available statistics related to physical activities sector and research works linked to teenager's mobility led us to question whether some factors impact differently boys and girls. Estimations resulting from a generalized ordinal logistic regression model developed using French National Travel Survey 2008 data show that effect of sex does not significantly differ neither according to "place of residence" nor according to "reference person's higher diploma", nor according to "income per consumption unit". Moreover, we show on the one hand that gender
\end{abstract}

David Sayagh $(\square)$

Ifsttar, Département Aménagement, Mobilités et Environnement (AME) Laboratoire Dynamiques économique et sociales des Transports (DEST) Cité Descartes, 14-20 bd Newton, 77447 Marne-la-Vallée Cedex 2, France e-mail : david.sayagh@ifsttar.fr gap towards likelihood of cycling reaches systematically its maximum level in areas classified as ZUS (Sensitive Urban Zones), and on the other hand that while during adolescence, this probability decreases for both boys and girls, the fall is much more pronounced and concentrated essentially between preadolescence and end of junior secondary school for girls.

Keywords bicycle adolescence - physical activity . gendered practice $\cdot Z U S$

\section{Introduction}

En Europe comme ailleurs, c'est principalement durant l'adolescence, période charnière de construction des identités sexuées [1], que les filles sont moins «physiquement actives » que les garçons [2] $]^{1}$. Si le phénomène préoccupe depuis près de vingt ans le gouvernement au Québec [3], ce n'est que très récemment qu'il a fait l'objet d'un chapitre dans un rapport ministériel en France $[4]^{2}$. L'initiative vise essentiellement à lutter contre les inégalités d'opportunités entre filles et garçons et la cristallisation des « natures "féminine" et "masculine" ». Une démarche qui implique de dénoncer les stéréotypes de sexe qui influencent les choix de pratiques physiques et se renforcent à travers elles en débouchant sur une féminité et une masculinité «normales/idéales-typiques » [5]. Aussi bien lorsqu'elles sont considérées en tant qu'activités sportives [4] que lorsqu'elles sont interrogées comme moyen de déplacement [6], les pratiques du vélo ne sont pas exclues par la tendance, et apparaissent particulièrement sexuées durant

\footnotetext{
${ }^{1}$ Les filles se déclarant systématiquement moins « physiquement actives » que les garçons dans les 41 pays sondés, par rapport à une recommandation de 60 minutes d'activité physique 5 ou 6 jours/semaine. À 11 ans, $19 \%$ des filles sont jugées suffisamment actives, contre $28 \%$ des garçons.

${ }^{2}$ Parmi les adolescent(e)s âgé(e)s entre 12 et 17 ans déclarant pratiquer le vélo comme activité sportive (désignant ici aussi bien les pratiques libres que les pratiques en club), $61 \%$ sont des garçons.
} 
l'adolescence. Alternatives parmi d'autres pour s'affranchir de la tutelle des instances traditionnelles d'encadrement, les mobilités à vélo constituent durant cette période un potentiel support de passage du monde familier au domaine public [7]. Véritable enjeu de santé public en termes de prévention de l'obésité [8], elles s'avèrent de surcroît déterminantes vis-à-vis des probabilités de pratique à l'âge adulte [9]. Durant l'adolescence, une partie non négligeable des pratiques du vélo n'ont pas de but et de fin en soi mais prennent la forme de mobilités circulaires, de « jeux en mouvement », de poursuites, ou autres « jeux de provocation » [10], qui pour être appréhendés, invitent à recourir à une acception dépassant la simple adéquation « origine-destination » des déplacements. Pour ce faire, nous proposons de partir du constat selon lequel tout(e) cycliste déclarant ne faire du vélo ni spécialement pour ses déplacements, ni spécialement pour faire de l'activité physique, se déplace, et fait de l'activité physique ${ }^{3}$ quand même. De ce fait, occulter une de ces deux dimensions revient, soit à oublier que lors de toute pratique du vélo, le corps se déplace dans un contexte donné, soit à nier que pour produire son propre déplacement, celui-ci s'active en entraînant une augmentation de sa dépense d'énergie. Ainsi, nous n'appréhendons pas les mobilités à vélo comme des moyens de réaliser des activités, mais comme des pratiques en soi. Soit toute pratique du vélo impliquant le corps comme principal moteur de sa propre mobilité. En nous émancipant délibérément de typologies vis-à-vis des finalités ou motifs d'usage, nous nous demandons si certains déterminants de l'ensemble des mobilités à vélo des adolescent(e)s impactent dans une mesure significativement différente les filles et les garçons. Après avoir identifié les variables explicatives les plus pertinentes au regard d'une bibliographie nécessairement pluridisciplinaire, nous testerons leur significativité dans un modèle de régression logistique réalisé à partir de l'Enquête nationale transports déplacements (ENTD) $2008^{4}$, grâce auquel nous effectuerons les estimations permettant de tester les hypothèses que nous aurons formulées.

\footnotetext{
${ }^{3}$ L'activité physique désigne tous les mouvements corporels produits par la contraction des muscles squelettiques entraînant une augmentation de la dépense énergétique au-dessus de la dépense de repos. Malgré les confusions encore fréquentes, le sport n'en est qu'un sous-ensemble, spécialisé, organisé, et facilité par les organisations sportives.

${ }^{4}$ Il est probable que le lecteur perçoive ici une contradiction dans le choix d'exploiter une enquête comme l'ENTD pour dépasser la simple adéquation « origine-destination » des déplacements, mais nous allons voir qu'une variable singulière s'avère particulièrement propice à cet effet.
}

\section{Un clivage sexué}

\subsection{Déterminé par les ressources économiques et culturelles des parents ?}

Le clivage observé durant l'adolescence vis-à-vis des activités physiques n'est pas seulement sexué. Les adolescent(e)s qui n'ont pas d'activité physique ou sportive en dehors des heures d'EPS (Éducation physique et sportive) sont avant tout des filles de milieux sociaux défavorisés, à la fois du point de vue des revenus et du capital scolaire de leurs parents [11]. Le niveau de diplôme s'avère être le facteur le plus discriminant : en France, si les adolescentes sont $57 \%$ à pratiquer au moins une fois par semaine quand leurs parents n'ont aucun diplôme, elles sont $87 \%$ à en faire autant lorsque l'un de leurs parents est diplômé du supérieur [12]. Dans la mesure où les mobilités à vélo constituent une activité physique typique, il paraît légitime de poser l'hypothèse selon laquelle celles-ci seraient influencées par ces mêmes déterminants socio-économiques et culturels, lesquels pourraient également avoir une influence différente sur les adolescentes et les adolescents. En outre, parce que l'usage du vélo implique l'exposition du corps des pratiquant(e)s dans des espaces variés, il convient également de se demander si certains facteurs territoriaux sont susceptibles d'impacter différemment les pratiques des filles et celles des garçons.

\subsection{Dépendant du lieu de résidence?}

Nombre de travaux indiquent que l'intersection des inégalités entre les sexes avec d'importantes inégalités sociales et territoriales, conduit à des inégalités renforcées [13]. Malgré un manque de données sexuées territorialisées déploré sur l'analyse des mobilités en milieu rural [14], des recherches mettent en évidence la prégnance particulière des stéréotypes de sexe dans ces environnements [13], lesquels pourraient représenter un frein aux pratiques des filles. A contrario, d'autres travaux, comme ceux de Raibaud [15], pointent aujourd'hui du doigt les aménagements et équipements publics des villes qui, tout en favorisant les pratiques urbaines des garçons, expliqueraient en contrepartie le « décrochage $»^{5}$ des filles à partir de la classe de sixième. En outre, Oppenchaim constate qu'après quatorze ans, les filles résidant en ZUS (Zones

\footnotetext{
${ }^{5}$ À partir de l'entrée en sixième, les filles disparaîtraient des équipements et espaces publics destinés aux loisirs et aux activités sportives des jeunes.
} 
urbaines sensibles) ${ }^{6}$ se voient attribuer par leurs parents, un traitement spécifique notamment caractérisé par un accompagnement des mobilités plus fréquent qu'auparavant [7]. Autant de raisons de penser qu'au même titre que la catégorie d'unité urbaine du lieu résidence, l'âge pourrait influencer en partie le clivage sexué des pratiques du vélo durant l'adolescence.

\subsection{Lié à l’âge ?}

Bien qu'à ce sujet, la plupart des études sur les pratiques d'activités physiques des adolescent(e)s se contentent de conclure à un déclin observé tant chez les garçons que chez les filles, certaines vont plus loin en révélant un écart qui se creuserait en raison d'un écroulement particulièrement précoce et prononcé des pratiques des filles [16]. D'une manière générale, les garçons continuent à jouer à des âges plus avancés que les filles [17], et les pratiques de la bicyclette ne sont pas exclues de cette tendance. Devaux [7] observe à ce titre que si durant « la préadolescence » (11-13 ans) les garçons apprennent progressivement à réaliser des pratiques d' « auto-mobilités », notamment à travers des « jeux en mouvement » [18] à vélo à partir desquels ils s'approprient l'espace public résidentiel, les filles investissent à l'inverse davantage la sphère domestique, en particulier au travers du développement d'une forte « culture de la chambre » [19] qui demeure prégnante durant la « fin des années collège » (14-16 ans).

En somme, la littérature collectée amène à penser que l'âge des adolescent(e)s, leur lieu de résidence (et notamment le fait de résider en ZUS) ainsi que les niveaux de revenu et de diplôme de leurs parents, devraient influencer le clivage sexué des pratiques du vélo au cours de l'adolescence. Après avoir vérifié qu'il existe un lien significatif entre ces variables et les pratiques des garçons d'une part, et celles des filles d'autre part, nous tenterons d'identifier à partir d'une méthode de régression logistique pas à pas, quelles variables impactent significativement différemment les filles et les garçons (avec un seuil de signification de 0,95 ).

\section{Méthodologie et résultats}

L'ENTD 2008 constitue sur le plan national, la base de données la plus complète pour caractériser les mobilités

\footnotetext{
${ }^{6}$ Les ZUS ont depuis été supprimées et remplacées par les Quartiers prioritaires de la politique de la ville (QPV) le 1er janvier 2015. Néanmoins, la base de données exploitée dans cet article ayant été produite antérieurement à ce changement, nous faisons le choix de maintenir l'ancienne appellation.
}

réalisées à vélo en rapport avec la géographie, ainsi que les activités économiques et sociales des membres du ménage. L'enquête a été réalisée en deux visites au domicile des ménages (France métropolitaine), en face-à-face avec l'intégralité des adultes, ainsi qu'un enfant âgé de 6 ans ou plus ${ }^{7}$, tiré de façon aléatoire parmi ceux du ménage. La variable expliquée est issue d'une séquence dédiée aux «pratiques de mobilité et de loisirs " 8 , abordée lors de la première visite. Elle établit trois degrés de pratique : «X se déplace à vélo »\{Oui, « régulièrement » (toutes les semaines)/Oui, « occasionnellement »/Non, « jamais »\}, et s'avère particulièrement intéressante pour deux raisons. D’une part, parce qu'elle n'appréhende pas le vélo comme le moyen de réaliser une activité mais comme une pratique en soi. D'autre part, parce qu'elle permet de faire un compromis dépassant la simple bi-catégorisation pratiquant/non-pratiquant. La nécessité de conserver des effectifs conséquents pour chaque modalité nous conduit à ne pas dépasser quatre catégories par classe pour le découpage des variables explicatives. C'est le cas de la variable « quartile de revenu par unité de consommation (UC) » du ménage (Q1/Q2/Q3/Q4) préférée à la variable « revenu de la personne de référence » pour mieux refléter le niveau de ressource économique du ménage. Afin d'obtenir des classes homogènes, nous découpons la variable « diplôme le plus élevé de la Personne de référence » $(\mathrm{PR})$ du ménage en regroupant les niveaux I, II et III définis par l'Insee $[10]^{9}$ dans une seule et même classe : « $\geq$ bac $+2 »$. Nous décidons ensuite de nous appuyer sur une définition couramment utilisée par l'Insee et le ministère de l'Éducation nationale pour décrire la population des nondiplômés. Nous considérons ainsi comme personne sans diplôme tout individu dont la formation initiale n'est pas validée au-delà de la scolarité obligatoire qu'il possède ou non le Certificat d'études primaires ou le Brevet des collèges [20]. Nous obtenons en somme la classification suivante : « $\geq$ bac $+2 » / \ll<\mathrm{bac}+2 » / \ll$ aucun diplôme ». Aussi, nous construisons nos classes d'âge en nous basant notamment sur les trois phases identifiées par Devaux à partir d'une analyse des mobilités quotidiennes d'adolescents ruraux 10 . Celles-ci nous conduisent à obtenir un échantillon constitué de 1649 garçons et 1429 filles, découpé en intervalles irréguliers : «11-13 ans », «14-16 ans » et «17-18

\footnotetext{
${ }^{7}$ Soit scolarisé, soit travailleur.

${ }^{8}$ Chapitre introduit par l'enquêteur de la manière suivante « Nous allons maintenant poser quelques questions sur les pratiques de mobilité et de loisirs de (prénom) ».

${ }^{9}$ Les niveaux II et I correspondent aux diplômes de $2^{\mathrm{e}}$ ou $3^{\mathrm{e}}$ cycle universitaire (licence, maîtrise, master, DEA, DESS, doctorat) ou diplômes de grande école. Le niveau III aux diplômes de niveau Bac+ 2 (DUT, BTS...) : $\mathrm{http}: / / \mathrm{www}$.insee.fr/fr/methodes/default.asp?page=definitions/niveaudiplome.htm
} 
ans ». Enfin, les éléments bibliographiques rassemblés nous amènent à sélectionner deux variables relatives au lieu de résidence. La première, "catégorie d'unité urbaine de la résidence principale » fait l'objet d'un découpage standard : Ville centre/Banlieue/Commune rurale ${ }^{10}$, la deuxième fait simplement la distinction ZUS/non ZUS.

\subsection{Des pratiques régulières fortement sexuées}

Avant de tester la dépendance de chacune des variables sélectionnées avec les pratiques des filles $(n=1429)$ d'une part, et celles des garçons $(n=1649)$ d'autre part, un premier croisement test nous permet de vérifier qu'il existe bien un lien hautement significatif $(p<0,0001)$ au test du khi-deux entre la variable « sexe » et le fait de déclarer pratiquer le vélo « régulièrement » (toutes les semaines), « occasionnellement $»$ ou « jamais ». Ainsi, quand près de $31 \%$ des garçons âgés entre 11 et 18 ans déclarent une pratique régulière, c'est le cas de $12 \%$ des filles du même âge. Aussi, alors que les garçons sont près de $31 \%$ à ne jamais faire de vélo, c'est le cas de près de $48 \%$ des filles, lesquelles sont en proportion légèrement plus nombreuses à déclarer une pratique occasionnelle ( $40 \%$ contre $38 \%$ pour les garçons) (Tableau 1). Si le clivage sexué s'observe tant pour la pratique régulière que pour l'absence de pratique, on ne peut pas en dire autant pour l'usage occasionnel, ce qui ne devrait pas nous empêcher d'observer des variations d'effet en fonction des modalités des variables explicatives retenues.

À titre exploratoire, il s'agit désormais de vérifier que les cinq variables retenues s'avèrent bien significatives au test du khi-deux vis-à-vis des pratiques des filles d'une part, et de celles des garçons (11-18 ans) d'autre part. Pour ce faire, nous produisons des tableaux croisés distincts entre filles

Tableau 1 Pratiques du vélo des adolescent(e)s

(11-18 ans) selon le sexe

\begin{tabular}{lcccr}
\hline X se déplace à vélo & Garçons & $\mathbf{N}$ & Filles & $\mathbf{N}$ \\
\hline régulièrement & $31 \%$ & 508 & $12 \%$ & 172 \\
occasionnellement & $38 \%$ & 632 & $40 \%$ & 570 \\
jamais & $31 \%$ & 509 & $48 \%$ & 687 \\
Total & & 1649 & & 1429 \\
\hline
\end{tabular}

\footnotetext{
${ }^{10} \mathrm{Si}$ une commune représente plus de $50 \%$ de la population d'une unité urbaine, elle est seule ville centre. Dans le cas contraire, toutes les communes qui ont une population supérieure à la moitié de celle de la commune la plus importante, ainsi que cette dernière, sont ville centre. Les communes urbaines qui ne sont pas des villes centres constituent la banlieue de l'unité urbaine. Les communes rurales sont celles qui n'appartiennent pas à une unité urbaine.
}

et garçons, ce qui nous permet aussi de rendre compte des effectifs pour chaque modalité.

\subsection{Des pratiques moins fréquentes lorsque la personne de référence du ménage ne possède aucun diplôme}

Pour les garçons comme pour les filles, nous relevons d'une part un lien hautement significatif (respectivement $\mathrm{p}=0,0007$ et $\mathrm{p}<0,0001)$ entre les variables « diplôme le plus élevé de la $\mathrm{PR}$ » et « $\mathrm{X}$ se déplace à vélo », et d'autre part des pratiques régulières et occasionnelles du vélo qui apparaissent moins fréquentes lorsque la « PR du ménage ne possède aucun diplôme ». Ainsi, quand $26 \%$ des garçons et $43 \%$ des filles dont la « PR possède un diplôme supérieur ou égal à bac $+2 »$ ne font jamais de vélo, c'est le cas de $39 \%$ des garçons et de $60 \%$ des filles dont la personne de référence n'est titulaire d'aucun diplôme (Tableaux 2 et 3 ).

\subsection{Des pratiques moins fréquentes pour les plus défavorisé(e)s}

Pour les garçons comme pour les filles, nous notons d'une part un lien hautement significatif (respectivement $p<0,0001$ et $p=0,0004)$ entre les variables « quartile de revenu simulé par $\mathrm{UC}$ » et « $\mathrm{X}$ se déplace à vélo », et d'autre part, que le fait de ne jamais faire de vélo est plus fréquent lorsque ceux/celles-ci dépendent du $1^{\text {er }}$ quartile de

Tableau 2 Pratiques du vélo des garçons (11-18 ans) en fonction du diplôme le plus élevé de la PR

\begin{tabular}{|c|c|c|c|}
\hline $\begin{array}{l}\text { Diplôme le plus } \\
\text { élevé de la PR }\end{array}$ & $\mathrm{X}$ se déplace à vélo & Garçons & $\mathbf{N}$ \\
\hline \multirow{3}{*}{ aucun diplôme* } & régulièrement & $27 \%$ & 103 \\
\hline & occasionnellement & $34 \%$ & 131 \\
\hline & jamais & $39 \%$ & 150 \\
\hline \multirow{3}{*}{$<\mathrm{bac}+2$} & régulièrement & $32 \%$ & 255 \\
\hline & occasionnellement & $38 \%$ & 300 \\
\hline & jamais & $30 \%$ & 238 \\
\hline \multirow{3}{*}{$\geq \mathrm{bac}+2$} & régulièrement & $32 \%$ & 150 \\
\hline & occasionnellement & $43 \%$ & 201 \\
\hline & jamais & $26 \%$ & 121 \\
\hline \multicolumn{2}{|l|}{ Total } & & 1649 \\
\hline \multicolumn{2}{|l|}{$\mathrm{p}$} & & 0,0007 \\
\hline
\end{tabular}


Tableau 3 Pratiques du vélo des filles (11-18 ans) en fonction du diplôme le plus élevé de la PR

\begin{tabular}{|c|c|c|c|}
\hline $\begin{array}{l}\text { Diplôme le plus } \\
\text { élevé de la PR }\end{array}$ & $\mathrm{X}$ se déplace à vélo & Filles & $\mathbf{N}$ \\
\hline \multirow{3}{*}{ aucun diplôme* } & régulièrement & $9 \%$ & 32 \\
\hline & occasionnellement & $32 \%$ & 112 \\
\hline & jamais & $60 \%$ & 212 \\
\hline \multirow{3}{*}{$<\mathrm{bac}+2$} & régulièrement & $13 \%$ & 88 \\
\hline & occasionnellement & $42 \%$ & 287 \\
\hline & jamais & $45 \%$ & 309 \\
\hline \multirow{3}{*}{$\geq \mathrm{bac}+2$} & régulièrement & $13 \%$ & 52 \\
\hline & occasionnellement & $44 \%$ & 171 \\
\hline & jamais & $43 \%$ & 166 \\
\hline \multicolumn{2}{|l|}{ Total } & & 1429 \\
\hline \multicolumn{2}{|l|}{$\mathrm{p}$} & \multicolumn{2}{|r|}{$<0,0001$} \\
\hline \multicolumn{4}{|c|}{$\begin{array}{l}\text { *Lorsque la formation initiale n'est pas validée au-delà de la scolarité obligatoire, } \\
\text { qu'il y ait possession ou non du Certificat d'études primaires ou du Brevet des } \\
\text { collèges. }\end{array}$} \\
\hline
\end{tabular}

Tableau 4 Pratiques du vélo des garçons (11-18 ans) en fonction du quartile de revenu simulé/UC*

\section{Quartile de revenu $\mathrm{X}$ se déplace à vélo Garçons $\quad \mathrm{N}$ simulé par UC}

\begin{tabular}{|c|c|c|c|}
\hline & régulièrement & $26 \%$ & 118 \\
\hline \multirow[t]{3}{*}{ Q1 } & occasionnellement & $32 \%$ & 143 \\
\hline & jamais & $43 \%$ & 193 \\
\hline & régulièrement & $32 \%$ & 138 \\
\hline \multirow[t]{3}{*}{ Q2 } & occasionnellement & $42 \%$ & 181 \\
\hline & jamais & $27 \%$ & 116 \\
\hline & régulièrement & $34 \%$ & 130 \\
\hline \multirow[t]{3}{*}{ Q3 } & occasionnellement & $37 \%$ & 144 \\
\hline & jamais & $29 \%$ & 111 \\
\hline & régulièrement & $33 \%$ & 122 \\
\hline \multirow[t]{2}{*}{ Q4 } & occasionnellement & $44 \%$ & 164 \\
\hline & jamais & $24 \%$ & 89 \\
\hline Total & & & 1649 \\
\hline $\mathrm{p}$ & & & 0,0001 \\
\hline
\end{tabular}

revenu (les $25 \%$ les moins favorisés) plutôt que des autres catégories. Ainsi, près de $58 \%$ des filles et $43 \%$ des garçons appartenant au $1^{\mathrm{er}}$ quartile ne font jamais de vélo alors que ce n'est le cas que pour $44 \%$ des filles et $24 \%$ des garçons dépendant du $4^{\mathrm{e}}$ quartile. Aussi, pouvons-nous observer que les filles comme les garçons dépendant du $1^{\mathrm{er}}$ quartile sont moins nombreu(x)ses à déclarer des pratiques régulières et occasionnelles que celles et ceux qui appartiennent au $4^{\mathrm{e}}$ quartile (Tableaux 4 et 5).

\subsection{Des pratiques plus fréquentes en commune rurale}

Pour les garçons comme pour les filles, nous notons un lien hautement significatif (respectivement $\mathrm{p}=0,0065$ et $\mathrm{p}$ $<0,0001)$ entre les variables « catégorie d'unité urbaine de la résidence principale » et « $\mathrm{X}$ se déplace à vélo ». Nous relevons que pour les adolescents et les adolescentes, la pratique régulière comme la pratique occasionnelle du vélo sont plus fréquentes en commune rurale, et ne diffèrent que très peu entre les catégories banlieue et ville centre (avec un écart n'excédant jamais 1 point). Ainsi, alors que $33 \%$ des garçons résidant en commune rurale déclarent pratiquer régulièrement le vélo, ceux qui résident en ville centre et en banlieue ne sont respectivement que $28 \%$ et $29 \%$ à déclarer

Tableau 5 Pratiques du vélo des filles (11-18 ans) en fonction du quartile de revenu simulé/UC*

\begin{tabular}{|c|c|c|c|}
\hline $\begin{array}{l}\text { Quartile de revenu } \\
\text { simulé par UC }\end{array}$ & $\mathrm{X}$ se déplace à vélo & Filles & $\mathbf{N}$ \\
\hline & régulièrement & $11 \%$ & 45 \\
\hline \multirow[t]{3}{*}{ Q1 } & occasionnellement & $32 \%$ & 134 \\
\hline & jamais & $58 \%$ & 243 \\
\hline & régulièrement & $11 \%$ & 42 \\
\hline \multirow[t]{3}{*}{ Q2 } & occasionnellement & $44 \%$ & 175 \\
\hline & jamais & $45 \%$ & 179 \\
\hline & régulièrement & $14 \%$ & 50 \\
\hline \multirow[t]{3}{*}{ Q3 } & occasionnellement & $43 \%$ & 148 \\
\hline & jamais & $43 \%$ & 149 \\
\hline & régulièrement & $13 \%$ & 35 \\
\hline \multirow[t]{2}{*}{ Q4 } & occasionnellement & $43 \%$ & 113 \\
\hline & jamais & $44 \%$ & 116 \\
\hline Total & & & 1429 \\
\hline $\mathrm{p}$ & & & 0,0004 \\
\hline
\end{tabular}




\begin{tabular}{|c|c|c|c|}
\hline $\begin{array}{l}\text { Catégorie d'unité } \\
\text { urbaine }\end{array}$ & $\mathrm{X}$ se déplace à vélo & Garçons & $\mathbf{N}$ \\
\hline \multirow{3}{*}{ banlieue } & régulièrement & $29 \%$ & 182 \\
\hline & occasionnellement & $37 \%$ & 235 \\
\hline & jamais & $34 \%$ & 213 \\
\hline \multirow{3}{*}{ commune rurale* } & régulièrement & $33 \%$ & 195 \\
\hline & occasionnellement & $42 \%$ & 247 \\
\hline & jamais & $25 \%$ & 150 \\
\hline \multirow{3}{*}{ ville centre } & régulièrement & $28 \%$ & 86 \\
\hline & occasionnellement & $37 \%$ & 114 \\
\hline & jamais & $35 \%$ & 108 \\
\hline \multicolumn{2}{|l|}{ Total } & \multicolumn{2}{|r|}{1530} \\
\hline \multicolumn{2}{|l|}{$\mathrm{p}$} & \multicolumn{2}{|r|}{0,0065} \\
\hline
\end{tabular}

Tableau 7 Pratiques du vélo des filles (11-18 ans) en fonction de la catégorie d'unité urbaine de leur lieu de résidence

\begin{tabular}{|c|c|c|c|}
\hline $\begin{array}{l}\text { Catégorie d'unité } \\
\text { urbaine }\end{array}$ & $X$ se déplace à vélo & Filles & $\mathbf{N}$ \\
\hline & régulièrement & $11 \%$ & 55 \\
\hline \multirow[t]{3}{*}{ banlieue } & occasionnellement & $35 \%$ & 174 \\
\hline & jamais & $54 \%$ & 273 \\
\hline & régulièrement & $14 \%$ & 74 \\
\hline \multirow[t]{3}{*}{ commune rurale* } & occasionnellement & $46 \%$ & 243 \\
\hline & jamais & $40 \%$ & 207 \\
\hline & régulièrement & $12 \%$ & 32 \\
\hline \multirow[t]{2}{*}{ ville centre } & occasionnellement & $35 \%$ & 98 \\
\hline & jamais & $53 \%$ & 148 \\
\hline \multicolumn{2}{|l|}{ Total } & & 1304 \\
\hline \multicolumn{2}{|l|}{$\mathrm{p}$} & & $<0,0001$ \\
\hline
\end{tabular}

en faire autant. Et, alors que $14 \%$ des filles résidant en commune rurale déclarent pratiquer régulièrement le vélo, celles qui résident en ville centre et en banlieue ne sont respectivement que $12 \%$ et $11 \%$ à déclarer en faire autant (Tableaux 6 et 7).
Tableau 8 Pratiques du vélo des garçons (11-18 ans) en ZUS ou non

\begin{tabular}{llrr}
\hline ZUS & X se déplace à vélo & Garçons & N \\
\hline & régulièrement & $32 \%$ & 495 \\
non ZUS & occasionnellement & $39 \%$ & 604 \\
& jamais & $30 \%$ & 472 \\
& & & \\
& régulièrement & $17 \%$ & 13 \\
ZUS & occasionnellement & $36 \%$ & 28 \\
& jamais & $47 \%$ & 37 \\
\hline \multirow{2}{*}{ Total } & & & 1649 \\
$\mathrm{p}$ & & & 0,0017 \\
\hline
\end{tabular}

\begin{tabular}{|c|c|c|c|}
\hline \multicolumn{4}{|c|}{$\begin{array}{l}\text { Tableau } 9 \text { Pratiques du vélo des filles (11-18 } \\
\text { ans) en ZUS ou non }\end{array}$} \\
\hline ZUS & $\mathrm{X}$ se déplace à vélo & Filles & $\mathbf{N}$ \\
\hline non ZUS & $\begin{array}{l}\text { régulièrement } \\
\text { occasionnellement } \\
\text { jamais }\end{array}$ & $\begin{array}{l}13 \% \\
41 \% \\
47 \%\end{array}$ & $\begin{array}{l}171 \\
551 \\
632\end{array}$ \\
\hline ZUS & $\begin{array}{l}\text { régulièrement } \\
\text { occasionnellement } \\
\text { jamais }\end{array}$ & $\begin{array}{r}1 \% \\
25 \% \\
73 \%\end{array}$ & $\begin{array}{r}1 \\
19 \\
55\end{array}$ \\
\hline $\begin{array}{l}\text { Total } \\
\mathrm{p}\end{array}$ & & & $\begin{array}{r}1429 \\
<0,0001\end{array}$ \\
\hline
\end{tabular}

\subsection{Des pratiques moins fréquentes en ZUS}

Pour les garçons comme pour les filles, nous notons un lien hautement significatif (respectivement $\mathrm{p}=0,0017$ et $p<0,0001)$ entre les variables «ZUS » et « $X$ se déplace à vélo ». Nous relevons que pour les adolescents et les adolescentes, la pratique régulière comme la pratique occasionnelle du vélo sont moins fréquentes en ZUS qu'ailleurs. Ainsi, l'absence de pratique concerne $47 \%$ des garçons et $73 \%$ des filles résidant en ZUS et seulement $30 \%$ des garçons et $47 \%$ des filles qui résident hors ZUS (Tableaux 8 et 9 ).

\subsection{Une diminution des pratiques en fonction de l'âge}

Pour les garçons comme pour les filles, nous notons un lien hautement significatif $(p<0,0001)$ entre les variables 
Tableau 10 Pratiques du vélo des garçons (11-18 ans) en fonction de leur classe d'âge

Classe d'âge $X$ se déplace à vélo Garçons $\quad N$

\begin{tabular}{|c|c|c|c|}
\hline \multirow{3}{*}{ 11-13ans } & régulièrement & $40 \%$ & 240 \\
\hline & occasionnellement & $39 \%$ & 236 \\
\hline & jamais & $21 \%$ & 124 \\
\hline \multirow{3}{*}{ 14-16ans } & régulièrement & $31 \%$ & 184 \\
\hline & occasionnellement & $39 \%$ & 237 \\
\hline & jamais & $30 \%$ & 183 \\
\hline \multirow{3}{*}{ 17-18ans } & régulièrement & $19 \%$ & 84 \\
\hline & occasionnellement & $36 \%$ & 159 \\
\hline & jamais & $45 \%$ & 202 \\
\hline \multicolumn{2}{|l|}{ Total } & & 1649 \\
\hline \multicolumn{2}{|l|}{$\mathrm{p}$} & \multicolumn{2}{|r|}{$<0,0001$} \\
\hline
\end{tabular}

Tableau 11 Pratiques du vélo des filles (11-18 ans) en fonction de leur classe d'âge

\section{Classe d'âge $X$ se déplace à vélo Filles $\quad N$}

\begin{tabular}{|c|c|c|c|}
\hline \multirow{3}{*}{ 11-13ans } & régulièrement & $19 \%$ & 99 \\
\hline & occasionnellement & $50 \%$ & 256 \\
\hline & jamais & $31 \%$ & 159 \\
\hline \multirow{3}{*}{ 14-16ans } & régulièrement & $10 \%$ & 52 \\
\hline & occasionnellement & $38 \%$ & 210 \\
\hline & jamais & $52 \%$ & 285 \\
\hline \multirow{3}{*}{ 17-18ans } & régulièrement & $6 \%$ & 21 \\
\hline & occasionnellement & $28 \%$ & 104 \\
\hline & jamais & $66 \%$ & 243 \\
\hline \multicolumn{2}{|l|}{ Total } & & 1429 \\
\hline \multicolumn{2}{|l|}{$\mathrm{p}$} & \multicolumn{2}{|r|}{$<0,0001$} \\
\hline
\end{tabular}

« classe d'âge » et « $X$ se déplace à vélo ». D'une manière générale, nous constatons également que la pratique régulière et la pratique occasionnelle décroissent en fonction de la montée en âge. Ainsi, alors que l'absence de pratique ne concerne que $21 \%$ des garçons et $31 \%$ des filles âgé(e)s entre 11 et 13 ans, elle rassemble $45 \%$ des garçons et $66 \%$ des filles âgé(e)s entre 17 et 18 ans (Tableaux 10 et 11).

\section{Justification et procédure du modèle utilisé}

Nous savons désormais qu'il existe un lien hautement significatif $(\mathrm{p}<0,01)$ au test du khi-deux entre la variable « sexe » et le fait de faire du vélo, variable à expliquer pour laquelle les cinq variables explicatives étudiées se sont également avérées significatives. Néanmoins, les tests réalisés ne permettent pas de contrôler les effets concomitants des variables testées, toutes susceptibles d'être partiellement corrélées aux précédentes et de jouer un rôle également dans le degré de pratique du vélo. La signification statistique observée est donc à prendre avec réserve avant d'être validée par un modèle de régression logistique, lequel va nous permettre de repérer quelle(s) variable(s) explicative(s) font significativement varier les effets de la variable « sexe ». Nous souhaitons expliquer la variable « $\mathrm{X}$ se déplace à vélo » \{Oui, « régulièrement» (toutes les semaines)/Oui, « occasionnellement »/Non, « jamais »\}. Dans la mesure où l'ordre des catégories n'est pas discutable, la plupart des auteurs recommandent l'utilisation de modèles de régression ordinaux, qui comportent moins de paramètres et sont plus simples à interpréter qu'un modèle de régression polytomique nominal [21]. Le modèle ordinal est basé sur les probabilités de distribution cumulées, soit celles de réalisation de $Y \leq i$, sa forme est la suivante :

$P(Y \leq i)=F\left(\alpha_{\mathrm{i}}+X_{\mathrm{y}}\right) 1<i<k$ où $\alpha 1, \ldots \ldots, \alpha k$ sont les k constantes et $Y$ le vecteur des autres paramètres, ceux correspondant aux variables explicatives. Parce que les modalités introduites pour représenter les variables explicatives ne sont pas indépendantes (leur somme vaut 1 quel que soit l'individu i), la procédure implique de définir des modalités de référence pour estimer les $\mathrm{k}$ coefficients. Pour faciliter l'analyse des pratiques des filles, nous choisissons « Garçons » comme modalité de référence de la variable « sexe ». Pour simplifier la comparaison entre les PR non diplômées et les deux autres classes de diplômées, nous désignons comme référence de la variable « diplôme le plus élevé de la PR du ménage », la modalité « aucun diplôme ». Pour les variables restantes, nous choisissons les modalités rassemblant le plus d'effectifs. Nous obtenons au final les modalités de référence comme suit :

- Sexe : garçons

- Diplôme le plus élevé de la PR du ménage : aucun diplôme

- Classe d'âge : 11-13 ans

- ZUS : NON ZUS

- Catégorie d'unité urbaine de la résidence principale: commune rurale

- Quartile de revenu par unité de consommation du ménage : Q1 


\begin{tabular}{|lrrrr|}
\hline Tableau 12 & Analyse selon la statistique de Wald & \\
\hline Effet & $\begin{array}{c}\text { Ddl } \\
\text { Statistique } \\
\text { de }\end{array}$ & Wr $>$ Khi-2 \\
\hline Sexe & 1 & 146,4 & $<0,0001$ \\
Classe d'âge & 2 & 120,1 & $<0,0001$ \\
Catégorie d'unité urbaine & 2 & 32,3 & $<0,0001$ \\
Revenu simulé par UC & 3 & 23,6 & $<0,0001$ \\
ZUS & 1 & 16,4 & $<0,0001$ \\
Diplôme le plus élevé de la PR & 2 & 2,7 & 0,2572 \\
\hline
\end{tabular}

Tableau 13 Tests du rapport de vraisemblance et du score

\begin{tabular}{lrrr} 
Tests & Khi-2 & Ddl & Pr $>$ Khi-2 \\
\hline Score & 2,68 & 2 & $>0,25$ \\
Rapport de vraisemblance & 2,70 & 2 & $>0,25$ \\
\hline
\end{tabular}

H0 : modèle avec la constante et toutes les variables à l'exception de la variable « diplôme 》.

H1 : modèle avec toutes les variables explicatives.

$\mathrm{Si}$ Ho est vraie, les statistiques des tests doivent suivre asymptotiquement un Khideux à 2 degrés de liberté. Ici, les valeurs 2,68 et 2,7 ont plus de $25 \%$ de chance d'être dépassées. On accepte donc la validité de Ho au seuil de $5 \%$.

\subsection{Sélection du modèle et analyse des effets}

Nous commençons par tester l'ensemble des variables explicatives sans effets d'interactions. Afin de retenir les variables les plus corrélées avec la variable à expliquer, et les moins corrélées entre elles [22], nous nous appuyons sur une procédure de sélection descendante, partant du modèle complet, pour éliminer pas à pas les variables non significatives au sens du test de Wald (seuil à $5 \%$ ). Le test global Beta $=0$ et le test de score pour l'hypothèse des cotes proportionnelles du modèle apparaissent hautement significatifs $(\mathrm{p}<0,0001)$. Par ordre de contribution décroissante, les effets « sexe, » " classe d'âge », " catégorie d'unité urbaine », " revenu simulé par $\mathrm{UC} »$, et «ZUS » s'avèrent hautement significatifs $(\mathrm{p}<0,01)$ (Tableau 12). Ce n'est pas le cas de l'effet «Diplôme le plus élevé de la $\mathrm{PR} »(\mathrm{p}=0,2572)$, que nous retirons du modèle suite aux tests du rapport de vraisemblance et du score d'une part (Tableau 13), et à la comparaison des critères de Schwartz (SC) et d'Akaike (AIC) des modèles avec et sans la variable en question d'autre part (Tableau 14).

L'absence de quasi-colinéarité entre les variables explicatives choisies est vérifiée afin de ne pas fausser
Tableau 14 Critères de Schwartz (SC) et d'Akaike (AIC) des différents modèles emboîtés (sans interaction)

\begin{tabular}{|c|c|c|c|}
\hline Effet & Ddl & AIC & SC \\
\hline Sexe & 1 & 5886,3 & 5904,1 \\
\hline Classe d'âge & 2 & 5754,4 & 5784,2 \\
\hline Catégorie d'unité urbaine & 2 & 5720,8 & 5762,4 \\
\hline Revenu simulé par UC & 3 & 5682,8 & 5742,3 \\
\hline ZUS & 1 & 5667,6 & 5733,1 \\
\hline Diplôme le plus élevé de la PR & 2 & 5668,9 & 5746,2 \\
\hline
\end{tabular}

Tableau 15 Critères de Schwartz (SC) et d'Akaike (AIC) des différents modèles emboîtés

\begin{tabular}{|c|c|c|c|c|}
\hline Effet & Ddl & AIC & SC & Pr $>$ Khi-2 \\
\hline Sexe & 1 & 5886,3 & 5904,1 & $<0,0001$ \\
\hline Classe d'âge & 2 & 5754,4 & 5784,2 & $<0,0001$ \\
\hline $\begin{array}{l}\text { Catégorie d'unité } \\
\text { urbaine }\end{array}$ & 2 & 5720,8 & 5762,4 & $<0,0001$ \\
\hline $\begin{array}{l}\text { Revenu simulé par } \\
\text { UC }\end{array}$ & 3 & 5682,8 & 5742,3 & $<0,0001$ \\
\hline ZUS & 1 & 5667,6 & 5733,1 & $<0,0001$ \\
\hline Sexe $\times$ Classe d'âge & 2 & 5653,6 & 5731 & 0,0002 \\
\hline Sexe $\times$ ZUS & 1 & 5649,7 & 5732,9 & 0,0170 \\
\hline $\begin{array}{l}\text { Sexe } \times \text { Revenu } \\
\text { simulé par UC }\end{array}$ & 3 & 5654,6 & 5767,6 & 0,2860 \\
\hline $\begin{array}{l}\text { Sexe } \times \text { Catégorie } \\
\text { d'unité urbaine }\end{array}$ & 2 & 5651,8 & 5753 & 0,5296 \\
\hline
\end{tabular}

les résultats ${ }^{11}$. Pour examiner ensuite si les différences de pratique selon le sexe varient en fonction des autres variables explicatives retenues, il convient d'étudier des effets d'interaction entre ces variables et la variable « sexe ». Dans cette perspective, une sélection par les critères de Schwartz (SC) et d'Akaike (AIC) des différents modèles emboîtés (Tableau 15) nous conduit à retenir un modèle contenant les interactions « sexe » $\times$ 《 classe d'âge » et « sexe » $x$ « ZUS », et excluant les interactions « sexe » $x$ « revenu simulé par $U C$ » et « sexe » $X$ 《 catégorie d'unité urbaine $»(\text { Tableau } 16)^{12}$. L'effet du sexe sur les pratiques

\footnotetext{
${ }^{11}$ Pour toutes les associations possibles de variables explicatives du modèle, le V de Cramer se situe dans l'intervalle $[-0,0079 ; 0,19780]$. Il n'y a donc pas de dépendance marquée.

${ }^{12} \mathrm{~A}$ titre indicatif, les interactions « revenu simulé par UC » $\times$ « catégorie d'unité urbaine, » et « revenu
} 
Tableau 16 Estimations par l'analyse du maximum de vraisemblance du modèle optimal

Paramètre

Ddl Valeur estimée Erreur type Khi-2 Wald Pr $>$ Khi-2

\begin{tabular}{|c|c|c|c|c|c|c|}
\hline Intercept* & $\begin{array}{l}\text { occasionnellement } \\
\text { régulièrement }\end{array}$ & $\begin{array}{l}1 \\
1\end{array}$ & $\begin{array}{r}1,2946 \\
-0,6745\end{array}$ & $\begin{array}{l}0,1138 \\
0,1113\end{array}$ & $\begin{array}{r}129,3118 \\
36,7593\end{array}$ & $\begin{array}{l}<0,0001 \\
<0,0001\end{array}$ \\
\hline Sexe & filles & 1 & $-0,5079$ & 0,1156 & 19,2962 & $<0,0001$ \\
\hline Classe d'âge & $\begin{array}{l}14-16 \text { ans } \\
17-18 \text { ans }\end{array}$ & $\begin{array}{l}1 \\
1\end{array}$ & $\begin{array}{l}-0,3043 \\
-0,8396\end{array}$ & $\begin{array}{l}0,1095 \\
0,1279\end{array}$ & $\begin{array}{r}7,7250 \\
43,0687\end{array}$ & $\begin{array}{r}0,0054 \\
<0,0001\end{array}$ \\
\hline Catégorie unité urbaine & $\begin{array}{l}\text { banlieue } \\
\text { ville centre }\end{array}$ & $\begin{array}{l}1 \\
1\end{array}$ & $\begin{array}{l}-0,5037 \\
-0,3259\end{array}$ & $\begin{array}{l}0,0884 \\
0,0957\end{array}$ & $\begin{array}{l}32,4717 \\
11,5895\end{array}$ & $\begin{array}{r}<0,0001 \\
0,0007\end{array}$ \\
\hline Revenu simulé par UC & $\begin{array}{l}\text { Q2 } \\
\text { Q3 } \\
\text { Q4 }\end{array}$ & $\begin{array}{l}1 \\
1 \\
1\end{array}$ & $\begin{array}{l}0,3769 \\
0,4092 \\
0,5716\end{array}$ & $\begin{array}{l}0,0931 \\
0,1016 \\
0,1091\end{array}$ & $\begin{array}{l}16,3870 \\
16,2333 \\
27,4555\end{array}$ & $\begin{array}{l}<0,0001 \\
<0,0001 \\
<0,0001\end{array}$ \\
\hline ZUS & & 1 & $-0,3350$ & 0,1995 & 2,8198 & 0,0931 \\
\hline $\begin{array}{l}\text { Sexe (filles) } \times \text { Classe d' } \\
\text { Sexe (filles) } \times \text { Classe d' } \\
\text { Sexe (filles) } \times \text { ZUS }\end{array}$ & $\begin{array}{l}\text { âge (14-16 ans) } \\
\text { âge (17-18 ans) }\end{array}$ & $\begin{array}{l}1 \\
1 \\
1\end{array}$ & $\begin{array}{l}-0,6828 \\
-0,4077 \\
-0,8166\end{array}$ & $\begin{array}{l}0,1638 \\
0,1965 \\
0,3423\end{array}$ & $\begin{array}{r}17,3809 \\
4,3043 \\
5,6918\end{array}$ & $\begin{array}{r}<0,0001 \\
0,0380 \\
0,0170\end{array}$ \\
\hline
\end{tabular}

*coefficient constant

du vélo, diffère donc selon le fait de résider en ZUS/ou non d'une part, et selon la classe d'âge d'autre part.

Une fois ces étapes de sélection achevées, nous pouvons nous pencher sur les estimations du modèle retenu (modèle optimal), au regard duquel le test de score pour l'hypothèse des cotes proportionnelles (Khi-deux de 61 pour $12 \mathrm{Ddl}$ ) $\mathrm{s}$ 'avère hautement significatif $(\mathrm{p}<0,0001)$. Nous constatons que par ordre de contribution décroissante, les effets « sexe », « classe d'âge, » « catégorie d'unité urbaine », « revenu simulé par UC », s'avèrent également hautement significatifs $(p<0,01)$. Si on ne peut pas en dire autant pour la variable «ZUS » qui ne s'avère significative qu'au seuil de $90 \%(\mathrm{p}=0,0931)$, celle-ci contribue à améliorer le modèle et s'avère significative en interaction avec la variable « sexe » $(\mathrm{p}=0,017)$ (Tableau 15). Concernant les interactions Sexe (fille) $\times$ Classe d'âge, nous relevons que si vis-à-vis de la modalité de référence "11-13 ans », l'effet de la modalité «17-18 ans » s'avère significatif

simulé par UC » $\times$ ZUS ont également été testées mais ne se sont pas avérées significatives (respectivement $p=0,2293$ et $p=0,3451)$. L'effet du « revenu par UC » sur les pratiques du vélo, ne diffère donc pas significativement selon le lieu de résidence. $(\mathrm{p}=0,038)$, c'est avec l'effet de la variable «14-16 ans » que l'interaction apparaît la plus significative $(\mathrm{p}<0,0001)$ (Tableau 16).

Le calcul des probabilités correspondant à tous les états des variables va nous permettre de fournir des informations plus concrètes et plus aisément interprétables.

\subsection{Estimations de probabilités}

Nous relevons d'abord que pour les garçons comme pour les filles, la probabilité de déclarer une pratique régulière chute entre les différentes « classes d'âge », augmente en fonction $\mathrm{du}$ " quartile de revenu », s'avère plus élevée en commune rurale, et généralement moins importante en banlieue qu'en ville centre. Bien que plus importante pour les garçons quelles que soient les interactions considérées entre ces variables, cette probabilité varie donc considérablement entre garçons d'une part, et entre filles d'autre part. Les différences selon le lieu de résidence sont particulièrement parlantes. À titre d'exemple, une fille âgée de 17 ou 18 ans dépendant du $3^{\mathrm{e}}$ quartile de revenu, résidant en banlieue-ZUS, a une probabilité 6 fois moins élevée de déclarer faire du vélo régulièrement qu'une fille de la 
même « classe d'âge, » dépendant du même quartile de revenu, mais résidant en commune rurale ( $2 \%$ contre $12 \%)$. En outre, nous notons que si entre les classes 11-13 ans et 17-18 ans, la probabilité de déclarer une pratique régulière se trouve généralement divisée par 2 chez les garçons, elle s'avère généralement divisée par 3 chez les filles, voire par 4 pour les résidentes de banlieue-ZUS appartenant aux $2^{\mathrm{e}}$ et $3^{\mathrm{e}}$ quartiles de revenu ( $8 \%$ contre $2 \%)$. En parallèle, nous constatons qu'alors que cette chute de probabilité apparaît relativement progressive entre les différentes classes d'âge chez les garçons, elle s'avère fortement concentrée entre les deux premières classes chez les filles (11-13 ans et14-16 ans), et évolue faiblement entre les classes 14-16 ans et 17-18 ans. Toujours vis-à-vis de la probabilité de déclarer une pratique régulière, nous notons ensuite que l'écart entre garçons et filles en ZUS est systématiquement supérieur ou égal à celui observé en zone non ZUS, quels que soient le « quartile de revenu » et la «classe d'âge » d'appartenance (Tableau 17).
Nous relevons ensuite que pour les garçons comme pour les filles, la probabilité de déclarer une pratique occasionnelle n'est pas nécessairement plus élevée en commune rurale. En outre, si cette probabilité a tendance à chuter entre les différentes classes d'âge et à augmenter entre les « quartiles de revenu » chez les filles, ce n'est pas toujours le cas pour les garçons chez qui la tendance est même parfois inversée, notamment lorsque ceux-ci résident en commune rurale. Aussi, si pour la classe 11-13 ans, la probabilité de déclarer une pratique occasionnelle n'est pas systématiquement plus élevée pour les garçons, le clivage sexué est nettement marqué quelles que soient les interactions considérées pour les classes 14-16 ans et 17-18 ans. D'ailleurs, comme cela est le cas vis-à-vis de la pratique régulière, l'écroulement de la probabilité de déclarer une pratique occasionnelle chez les filles s'avère fortement concentré entre les deux premières classes (11-13 ans-14-16 ans). Toujours vis-à-vis de la probabilité de déclarer une pratique occasionnelle, nous notons enfin, que

\begin{tabular}{|c|c|c|c|c|c|c|c|c|}
\hline revenu simulé par UC & catégorie unité urbaine & ZUS & $\begin{array}{l}\text { garçon } \\
\text { 11-13ans }\end{array}$ & $\begin{array}{l}\text { fille } \\
\text { 11-13ans }\end{array}$ & $\begin{array}{l}\text { garçon } \\
\text { 14-16ans }\end{array}$ & $\begin{array}{l}\text { fille } \\
\text { 14-16ans }\end{array}$ & $\begin{array}{l}\text { garçon } \\
\text { 17-18ans }\end{array}$ & $\begin{array}{l}\text { fille } \\
\text { 17-18ans }\end{array}$ \\
\hline \multirow{5}{*}{ Q1 } & banlieue & non ZUS & 0,24 & 0,16 & 0,19 & 0,06 & 0,12 & 0,05 \\
\hline & banlieue & ZUS & 0,18 & 0,06 & 0,14 & 0,02 & 0,09 & 0,02 \\
\hline & ville centre & non ZUS & 0,27 & 0,18 & 0,21 & 0,08 & 0,14 & 0,06 \\
\hline & ville centre & ZUS & 0,21 & 0,07 & 0,16 & 0,03 & 0,10 & 0,02 \\
\hline & commune rurale & non ZUS & 0,34 & 0,23 & 0,27 & 0,10 & 0,18 & 0,08 \\
\hline \multirow{5}{*}{ Q2 } & banlieue & non ZUS & 0,31 & 0,21 & 0,25 & 0,09 & 0,16 & 0,07 \\
\hline & banlieue & ZUS & 0,24 & 0,08 & 0,19 & 0,03 & 0,12 & 0,02 \\
\hline & ville centre & non ZUS & 0,35 & 0,24 & 0,28 & 0,11 & 0,19 & 0,08 \\
\hline & ville centre & ZUS & 0,28 & 0,09 & 0,22 & 0,04 & 0,14 & 0,03 \\
\hline & commune rurale & non ZUS & 0,43 & 0,31 & 0,35 & 0,14 & 0,24 & 0,11 \\
\hline \multirow{5}{*}{ Q3 } & banlieue & non ZUS & 0,32 & 0,22 & 0,25 & 0,09 & 0,17 & 0,07 \\
\hline & banlieue & ZUS & 0,25 & 0,08 & 0,20 & 0,03 & 0,13 & 0,02 \\
\hline & ville centre & non ZUS & 0,36 & 0,25 & 0,29 & 0,11 & 0,19 & 0,09 \\
\hline & ville centre & ZUS & 0,28 & 0,10 & 0,23 & 0,04 & 0,15 & 0,03 \\
\hline & commune rurale & non ZUS & 0,43 & 0,32 & 0,36 & 0,15 & 0,25 & 0,12 \\
\hline \multirow{5}{*}{ Q4 } & banlieue & non ZUS & 0,35 & 0,25 & 0,29 & 0,11 & 0,19 & 0,09 \\
\hline & banlieue & ZUS & 0,28 & 0,09 & 0,22 & 0,04 & 0,14 & 0,03 \\
\hline & ville centre & non ZUS & 0,39 & 0,28 & 0,32 & 0,13 & 0,22 & 0,10 \\
\hline & ville centre & ZUS & 0,32 & 0,11 & 0,26 & 0,04 & 0,17 & 0,03 \\
\hline & commune rurale & non ZUS & 0,47 & 0,35 & 0,40 & 0,17 & 0,28 & 0,13 \\
\hline
\end{tabular}




\begin{tabular}{|c|c|c|c|c|c|c|c|c|}
\hline revenu simulé par $\mathrm{UC}$ & catégorie unité urbaine & ZUS & $\begin{array}{l}\text { garçon } \\
\text { 11-13ans }\end{array}$ & $\begin{array}{l}\text { fille } \\
\text { 11-13ans }\end{array}$ & $\begin{array}{l}\text { garçon } \\
\text { 14-16ans }\end{array}$ & $\begin{array}{l}\text { fille } \\
\text { 14-16ans }\end{array}$ & $\begin{array}{l}\text { garçon } \\
\text { 17-18ans }\end{array}$ & $\begin{array}{l}\text { fille } \\
\text { 17-18ans }\end{array}$ \\
\hline \multirow{5}{*}{ Q1 } & banlieue & non ZUS & 0,45 & 0,41 & 0,43 & 0,27 & 0,37 & 0,23 \\
\hline & & ZUS & 0,43 & 0,24 & 0,40 & 0,11 & 0,32 & 0,09 \\
\hline & ville centre & non ZUS & 0,46 & 0,43 & 0,45 & 0,30 & 0,40 & 0,25 \\
\hline & & ZUS & 0,45 & 0,27 & 0,42 & 0,13 & 0,35 & 0,11 \\
\hline & commune rurale & non ZUS & 0,45 & 0,45 & 0,46 & 0,35 & 0,43 & 0,31 \\
\hline \multirow{5}{*}{ Q2 } & banlieue & non ZUS & 0,45 & 0,45 & 0,45 & 0,33 & 0,42 & 0,29 \\
\hline & & ZUS & 0,45 & 0,30 & 0,44 & 0,15 & 0,38 & 0,13 \\
\hline & ville centre & non ZUS & 0,44 & 0,45 & 0,46 & 0,36 & 0,44 & 0,31 \\
\hline & & ZUS & 0,46 & 0,33 & 0,45 & 0,18 & 0,40 & 0,15 \\
\hline & commune rurale & non ZUS & 0,42 & 0,45 & 0,44 & 0,40 & 0,45 & 0,37 \\
\hline \multirow{5}{*}{ Q3 } & banlieue & non ZUS & 0,45 & 0,45 & 0,46 & 0,33 & 0,42 & 0,29 \\
\hline & & ZUS & 0,45 & 0,31 & 0,44 & 0,16 & 0,38 & 0,13 \\
\hline & ville centre & non ZUS & 0,44 & 0,45 & 0,46 & 0,36 & 0,44 & 0,32 \\
\hline & & ZUS & 0,46 & 0,33 & 0,45 & 0,18 & 0,40 & 0,15 \\
\hline & commune rurale & non ZUS & 0,41 & 0,45 & 0,44 & 0,41 & 0,45 & 0,37 \\
\hline \multirow{5}{*}{ Q4 } & banlieue & non ZUS & 0,44 & 0,45 & 0,46 & 0,36 & 0,44 & 0,32 \\
\hline & & ZUS & 0,46 & 0,33 & 0,45 & 0,18 & 0,40 & 0,15 \\
\hline & ville centre & non ZUS & 0,43 & 0,46 & 0,45 & 0,38 & 0,45 & 0,35 \\
\hline & & ZUS & 0,45 & 0,36 & 0,46 & 0,20 & 0,42 & 0,17 \\
\hline & commune rurale & non ZUS & 0,39 & 0,44 & 0,43 & 0,42 & 0,46 & 0,39 \\
\hline
\end{tabular}

c'est systématiquement en ZUS que l'écart entre garçons et filles est le plus creusé au détriment des pratiques des filles (Tableau 18).

Aussi, nous notons que pour les garçons comme pour les filles, la probabilité de ne déclarer aucune pratique du vélo augmente selon l'âge, diminue en fonction du « quartile de revenu », est systématiquement plus élevée en banlieue qu'en ville centre, apparait sensiblement plus faible en commune rurale, et s'avère considérablement plus élevée en ZUS. Nous relevons par ailleurs que si cette probabilité est systématiquement plus importante chez les filles, c'est en ZUS et entre 14 et 16 ans, que l'écart avec les garçons est le plus creusé, quel que soit le « quartile de revenu » considéré. Ainsi, une fille âgée entre 14 et 16 ans, dépendant $\mathrm{du} 4^{\mathrm{e}}$ quartile de « revenu simulé par UC » $(25 \%$ les plus favorisés), résidant en ZUS, en ville centre, a $75 \%$ de chance de ne déclarer aucune pratique du vélo, soit une probabilité 2,6 fois supérieure à celle d'un garçon de la même « classe d'âge » et appartenant aux mêmes « catégories » $(29 \%)$ (Tableau 19).

\section{Discussion}

\subsection{Limites}

Avant de discuter ces résultats, prenons le temps de relever les principales limites de notre travail. Nous devons d'abord envisager que certains enquêtés aient pu omettre consciemment de déclarer certaines pratiques. Un problème qui se pose particulièrement pour les adolescent(e)s, qui se déplacent notamment dans l'optique de s'émanciper de la tutelle des adultes, présents au moment de l'enquête [7]. Par ailleurs, nous aurions pu faire le choix de développer davantage le fait que toutes classes d'âge confondues, c'est systématiquement lorsque les filles et les garçons résident en commune rurale et lorsque ceux/celles-ci dépendent du $4^{\mathrm{e}}$ quartile de revenu (les $25 \%$ les plus favorisés) que leur probabilité de déclarer une pratique régulière est la plus forte. Un constat qui appelle selon nous à redoubler d'effort afin de promouvoir et de démocratiser les pratiques urbaines du vélo des adolescent(e)s. En outre, nous devons souligner 


\begin{tabular}{|c|c|c|c|c|c|c|c|c|}
\hline revenu simulé par UC & catégorie unité urbaine & ZUS & $\begin{array}{l}\text { garçon } \\
\text { 11-13ans }\end{array}$ & $\begin{array}{l}\text { fille } \\
\text { 11-13ans }\end{array}$ & $\begin{array}{l}\text { garçon } \\
\text { 14-16ans }\end{array}$ & $\begin{array}{l}\text { fille } \\
\text { 14-16ans }\end{array}$ & $\begin{array}{l}\text { garçon } \\
\text { 17-18ans }\end{array}$ & $\begin{array}{l}\text { fille } \\
\text { 17-18ans }\end{array}$ \\
\hline \multirow{5}{*}{ Q1 } & banlieue & non ZUS & 0,31 & 0,43 & 0,38 & 0,67 & 0,51 & 0,72 \\
\hline & & ZUS & 0,39 & 0,70 & 0,46 & 0,86 & 0,59 & 0,89 \\
\hline & ville centre & non ZUS & 0,28 & 0,39 & 0,34 & 0,63 & 0,47 & 0,69 \\
\hline & & ZUS & 0,35 & 0,67 & 0,42 & 0,84 & 0,55 & 0,87 \\
\hline & commune rurale & non ZUS & 0,22 & 0,31 & 0,27 & 0,55 & 0,39 & 0,61 \\
\hline \multirow{5}{*}{ Q2 } & banlieue & non ZUS & 0,24 & 0,34 & 0,30 & 0,58 & 0,42 & 0,64 \\
\hline & & ZUS & 0,30 & 0,62 & 0,37 & 0,81 & 0,50 & 0,85 \\
\hline & ville centre & non ZUS & 0,21 & 0,30 & 0,26 & 0,54 & 0,38 & 0,60 \\
\hline & & ZUS & 0,27 & 0,58 & 0,33 & 0,79 & 0,46 & 0,83 \\
\hline & commune rurale & non ZUS & 0,16 & 0,24 & 0,20 & 0,46 & 0,30 & 0,52 \\
\hline \multirow{5}{*}{ Q3 } & banlieue & non ZUS & 0,23 & 0,33 & 0,29 & 0,57 & 0,41 & 0,64 \\
\hline & & ZUS & 0,30 & 0,61 & 0,36 & 0,81 & 0,49 & 0,85 \\
\hline & ville centre & non ZUS & 0,20 & 0,30 & 0,25 & 0,53 & 0,37 & 0,59 \\
\hline & & ZUS & 0,26 & 0,57 & 0,32 & 0,78 & 0,45 & 0,82 \\
\hline & commune rurale & non ZUS & 0,15 & 0,23 & 0,20 & 0,45 & 0,30 & 0,51 \\
\hline \multirow{5}{*}{ Q4 } & banlieue & non ZUS & 0,20 & 0,30 & 0,26 & 0,53 & 0,37 & 0,60 \\
\hline & & ZUS & 0,26 & 0,57 & 0,33 & 0,78 & 0,45 & 0,82 \\
\hline & ville centre & non ZUS & 0,18 & 0,26 & 0,23 & 0,49 & 0,33 & 0,55 \\
\hline & & ZUS & 0,23 & 0,53 & 0,29 & 0,75 & 0,41 & 0,80 \\
\hline & commune rurale & non ZUS & 0,13 & 0,20 & 0,17 & 0,41 & 0,26 & 0,47 \\
\hline
\end{tabular}

le fait que les enfants déscolarisés et sans emploi ne sont pas échantillonnés par l'ENTD. Un manque qui mériterait d'autant plus d'être corrigé que le taux de scolarisation et le pourcentage d'inactifs chez les 15-19 ans s'avèrent «préoccupants » en France [23]. Enfin, en se basant sur des mobilités réalisées plutôt que sur des différences en termes d'accès ou de potentialités réelles de pratique, nos résultats ne permettent pas en soi de traduire des inégalités d'opportunités entre filles et garçons. D'où la nécessité de mettre en exergue le caractère socialement construit du clivage sexué des pratiques du vélo durant l'adolescence.

\subsection{Les déterminants appelant des méthodes de recherches qualitatives}

\subsubsection{Des attitudes sexuées socialement construites}

Nos résultats indiquent que l'effet du « sexe » sur les pratiques du vélo des adolescent(e)s, ne diffère ni selon le
« diplôme le plus élevé de la PR », ni selon le « revenu simulé par UC ». Ils ne nous permettent donc pas de conforter l'hypothèse selon laquelle le clivage sexué des pratiques du vélo serait influencé par les mêmes variables renforçatrices du clivage sexué observable dans le domaine des activités physiques pris dans son ensemble. Un constat qui amène d'abord à émettre des réserves quant à la pertinence de formuler des hypothèses en appréhendant les mobilités à vélo comme des activités physiques quelconques. Néanmoins, nos résultats confirment également que, comme c'est le cas pour les activités physiques dans leur ensemble, le clivage sexué des pratiques du vélo est accentué au cours de l'adolescence. Aussi, comme on pouvait le supposer grâce aux travaux de Devaux 10, l'écart s'avère beaucoup plus prononcé et essentiellement concentré entre la "préadolescence » et la "fin des années collège », ce qui tend à conforter le constat d'une prégnance de la « culture de la chambre » chez les filles qui se prolonge durant la «fin des années collège ». Notons par ailleurs, que si le vélo représente dans un premier temps 
un support d'émancipation de la tutelle des parents en permettant aux adolescent(e)s de réaliser leurs premières « auto-mobilités », son image tend généralement à se dégrader au fur et à mesure que celle de la voiture se popularise [9]. Une dévalorisation moins prégnante chez les garçons [9], pour qui le vélo continue plus longtemps à jouer un rôle important, notamment vis-à-vis de leurs sociabilités ludiques inscrites dans l'espace public, lesquelles s'avèrent caractéristiques de la socialisation masculine à la mobilité 10. L'influence sociale des pairs joue un rôle déterminant vis-à-vis des pratiques du vélo [24], mais elle ne se traduit pas de la même manière pour les adolescentes et les adolescents. Une étude australienne révèle à ce sujet que si les garçons liés à plusieurs pairs à proximité de leur lieu de résidence sont plus susceptibles de faire du vélo de manière récréative et/ou utilitaire en semaine, les filles bénéficiant d'une situation comparable sont plus susceptibles de faire du vélo de manière récréative le week-end [25]. En outre, il est à noter que les filles sont socialisées dès le plus jeune âge à l'évitement du risque [26]. Tout en présentant par la suite un niveau d'internalisation des règles relatives à l'usage du vélo plus important que celui des garçons, elles font état d'une moindre propension que ces derniers à s'engager dans des comportements risqués à vélo [27]. Par conséquent, il est probable que les adolescentes soient plus susceptibles d'être découragées par des contextes de circulation encore peu adaptés aux pratiques du vélo, incitant, voire contraignant fréquemment les cyclistes à enfreindre une ou plusieurs règles du code de la route [28,29].

\subsubsection{Des répercussions différenciées sur le plan de la santé}

Il n'en demeure pas moins, que de manière générale, les cyclistes femmes (dans leur ensemble) privilégient les rues résidentielles et les trafics lents, peu denses, avec des routes offrant un maximum de séparation du reste du trafic [30,31,32]. Parce que comparé à la marche, le vélo peut toutefois faciliter la démarche des « traceuses » $[33]^{13}$, il constitue pour certaines un refuge permettant de « retrouver une liberté dans l'espace public » [34]. Mais cette opportunité n'est-elle pas l'apanage de celles qui ont gagné en assurance en continuant de pratiquer durant leur adolescence? Il n'en reste pas moins que les femmes seraient en proportion moins nombreuses que les hommes à prendre du plaisir à pratiquer le vélo [30], et qu'elles se déclarent également moins « à l'aise pour faire du vélo quotidiennement »(28,9\% contre $51,1 \%$ des hommes $)$

\footnotetext{
${ }^{13}$ Zaffran (2016) utilise le terme pour désigner la tendance des filles à se déplacer d'un lieu à un autre, en ne restant « pas longtemps au même endroit ».
}

[35]. De ces manières sexuées de pratiquer et de penser le vélo pourraient découler des répercussions différenciées sur le plan de la santé. En effet, nous savons notamment que la moindre pratique d'activité physique des filles est associée à des effets négatifs vis-à-vis de leurs aptitudes aérobies ainsi que de leurs prédispositions à l'obésité [36]. Or, la prévalence de l'obésité en France s'avère plus élevée chez les femmes $(15,7 \%)$ que chez les hommes $(14,3 \%)$ [37]. En outre, plusieurs travaux indiquent que les prises de « risques accidentels » et les « comportements imprudents » plus importants de la part des garçons et des hommes se traduisent par de plus grandes chances d'être tués et/ou gravement blessés à vélo $[38,39,40]^{14}$.

\subsubsection{Des pratiques éducatives sexuées notamment fondées sur la présumée particulière vulnérabilité des adolescentes}

Confrontés à la littérature disponible sur les processus de socialisation sexuée, nos résultats semblent par ailleurs conforter la thèse d'une forte remise en cause des pratiques éducatives des parents influencées par les stéréotypes de sexe associant «féminité » et «vulnérabilité 》. À ce titre, une étude australienne révèle que la perception de l'insécurité du quartier de résidence par les parents est associée à une restriction de l'activité physique (dont le vélo) des adolescentes supérieure à celle établie pour les adolescents du même âge [41]. Des traitements différenciés que les travaux de Rivière [42] permettent notamment d'associer aux premières transformations physiques connues par le corps des jeunes filles. C'est précisément lorsque celles-ci commencent à être dotées des « attributs corporels de la féminité » [43] que leur présumée vulnérabilité « féminine » permet aux parents de justifier la perte d'autonomie qui leur est alors imposée. Un constat global qui amène à se demander si certaines craintes sexuées sont directement associées aux pratiques du vélo des adolescentes.

\subsubsection{Des pratiques éducatives sexuées encore marquées par d'anciennes mises en garde de la médecine?}

Rappelons à ce sujet que la fragilité particulière des femmes a été médicalement démontrée jusqu'à la fin des années 1950, et que les pratiques de la bicyclette ont longtemps été placées au centre des débats. Assimilé au désir d'indépendance et d'égalité des femmes au début $\mathrm{du} \mathrm{XX}^{\mathrm{e}}$ siècle, le vélo est accusé par certains médecins

\footnotetext{
${ }^{14}$ Ceux-ci auraient 1,7 fois plus de chance d'être tués et 1,4 fois plus de risque d'être gravement blessés que les femmes cyclistes. Bien que les femmes auraient 2,43 fois plus de chance d'être blessées « toutes blessures confondues ».
} 
de l'époque, de porter atteinte au bon fonctionnement des organes reproducteurs, ou encore de posséder un potentiel masturbatoire [44]. Les adolescentes sont particulièrement visées. Y compris par les moins conservateurs de l'époque. C'est le cas du docteur O'Followell, auteur d'un ouvrage intitulé Bicyclettes et organes génitaux, dans lequel il recommande de «prescrire avec prudence l'usage du cyclisme chez les jeunes filles de 12 à 13 ans, au moment où la puberté s'établit. Il pourrait y avoir des inconvénients au point de vue de l'éveil de l'instinct génésique » [45]. Dans quelle mesure ces mises en garde sexuées contre la pratique de la bicyclette, alors qualifiée de «machine à stérilité » [46], animent-elles encore aujourd'hui les craintes de certains parents au point de les inciter à freiner les pratiques du vélo de leur(s) fille(s) durant l'adolescence ?

\subsubsection{L'espace public des ZUS, un terrain de jeu réservé aux garçons}

Bien qu'elle ne soit pas propre aux pratiques du vélo, Oppenchaim relève une intensification des accompagnements des mobilités des filles de ZUS après quatorze ans. Un phénomène qui serait notamment amplifié par une forte densité de population et l'interconnaissance entre les habitants de ces quartiers. Deux facteurs, qui en favorisant la circulation rapide de rumeurs de voisinage sur la « vertu amoureuse et/ou sexuelle » des filles, auraient notamment pour conséquence de nourrir chez les parents la crainte d'être soupçonnés de laxisme [7]. Dans ces quartiers plus qu'ailleurs, la voirie constitue un espace réservé aux hommes, où les jeunes filles doivent faire preuve de « réserve », leur visibilité dans l'espace public ne pouvant souvent être légitimée aux yeux des garçons que par des « visées sexuelles » [47]. Ainsi, pour protéger leur réputation (indissociable de celle de leur famille) et se prémunir contre d'inévitables agressions verbales et/ou physiques, celles-ci chercheraient à être le moins possible seules et le plus possible invisibles à l'extérieur [45]. En outre, au cœur d'une ZUS de Montpellier (La Mosson), Daude (2011) a observé que la peur de la «perte de la virginité » associée à la pratique de la gymnastique ou du vélo, s'avère extrêmement prégnante chez les jeunes filles issues de l'immigration maghrébine [48]. Un constat qui devrait selon nous inciter au renforcement des actions d'éducation à la sexualité dans les collèges et lycées de ces quartiers.

\subsubsection{Pratiques à caractère sportif : des freins multiples pour les filles}

Enfin, notons que les adolescent(e)s se représentent avant tout le vélo comme un « sport » [49]. Par conséquent nous pouvons penser que certains facteurs identifiés comme des obstacles à la pratique d'activités physiques et sportives des filles, participent également à renforcer le clivage sexué des pratiques du vélo durant l'adolescence. Parmi ces freins, nous pouvons citer le moindre sentiment de compétence des filles vis-à-vis de leurs capacités physiques et motrices, ainsi que la perception souvent négative de leur corps [50,51]. Nous pouvons également mentionner la moindre couverture médiatique du cyclisme féminin et la dépréciation des sportives en général [52]. Enfin, nous pouvons évoquer la croyance commune selon laquelle le fait de transpirer ne serait « pas très féminin ». Autant de pistes qui mériteraient d'être explorées via des approches qualitatives.

\section{Conclusion}

Nous avons vu que pour les garçons comme pour les filles, la probabilité de ne déclarer aucune pratique du vélo augmente selon l'âge, diminue en fonction du " quartile de revenu », est systématiquement plus élevée en banlieue qu'en ville centre, apparaît sensiblement plus faible en commune rurale, et s'avère considérablement plus élevée en ZUS. En outre, nous avons exposé deux principaux résultats, à savoir d'une part, que la probabilité de déclarer une pratique régulière ou occasionnelle du vélo décroît de manière plus prononcée et essentiellement concentrée entre la «préadolescence » et « la fin des années collège » chez les filles, et d'autre part, que c'est systématiquement en ZUS que le clivage s'avère le plus sexué. Tout en suggérant l'intérêt d'adopter une réflexion pluridisciplinaire, notre analyse dénote ensuite la nécessité de mettre à jour les inégalités que ce clivage recouvre. Notamment en étudiant les articulations entre les stéréotypes de sexe, les peurs et les pratiques éducatives parentales sexuées qui participent à le façonner. En outre, sachant qu'en France, les vélos écoles accueillent essentiellement des femmes originaires d'Afrique subsaharienne et du Maghreb, où la « culture vélo » est parfois inexistante et/ou souvent réservée aux hommes au point d'être parfois considérée comme incorrecte pour une femme [53], nos résultats devraient selon nous inciter à renforcer les actions de promotion du vélo auprès des adolescentes de ZUS.

\section{Bibliographie}

1. Chiland, $C$ (2014) « La construction de l'identité de genre à l'adolescence », Adolescence 32: 165-179

2. Currie, C, Zanotti, C, Morgan, A et al. (2012) Social determinants of health and well-being among young people: Health Behaviour in School-Aged Children (HBSC) study: international report from the 2009/2010 survey, Rapport de recherche pour la Health Policy for Children and Adolescents. 
3. Dugal, S (1998) Les filles c'est pas pareil, rapport sur la problématique des jeunes filles et la pratique de l'activité physique, Rapport de recherche pour le Gouvernement du Québec Kino-Québec, $36 \mathrm{p}$.

4. Naves, M-C, Octobre, S (2014) «Lutter contre les stéréotypes filles-garçons Un enjeu d'égalité et de mixité dès l'enfance », In : Naves, M-C, Wisnia-Weill, V (eds.), Inégalités et différences filles-garçons dans les pratiques sportives et culturelles des enfants et des adolescents, Commissariat général à la stratégie et à la prospective, Paris, pp 139-169.

5. Mennesson, C, Neyrand, G (2010) «La socialisation des filles et des garçons dans les pratiques culturelles et sportives », In : Octobre, S (eds.), Enfance et culture. Transmission, appropriation et représentation, DEPS, Ministère de la Culture et de la Communication, pp. 147-166.

6. Papon, F, de Solere, R (2010) « Les modes actifs: marche et vélo de retour en ville, La mobilité des Français », La Revue du CGDD, décembre, pp 65-82.

7. Oppenchaim, N (2012) «Mobilité quotidienne, socialisation et ségrégation: une analyse à partir des manières d'habiter des adolescents de zones urbaines sensibles», Thèse sous la direction de Francis Godard, Université Paris-Est, laboratoire Ville, Mobilité, Transport (LVMT), $591 \mathrm{p}$.

8. Menschik, D, Ahmed, S, Alexander, M-H, Blum, R-W (2008) «Adolescent physical activities as predictors of young adult weight », Archives of Pediatrics \& Adolescent Medicine 162: 23-28.

9. Underwood, S-K, Handy, S-L, Paterniti, D-A, Lee, A-E (2014) « Why do teens abandon bicycling? A retrospective look at attitudes and behaviors », Journal of Transport \& Health 1:17-24.

10. Devaux, J (2014) « Les trois âges de socialisation des adolescents ruraux. Une analyse à partir des mobilités quotidiennes ", Agora débats/jeunesses 68: 25-39.

11. Louveau, C (2004) «Pratiquer une activité physique ou sportive : persistance des inégalités parmi les femmes», Recherches feministes 17: 39-76.

12. Muller, L (2003) «La pratique sportive des jeunes dépend avant tout de leur milieu socioculturel», Insee première 932: $4 \mathrm{p}$.

13. Bousquet, D, Sabathier, R (2014) Combattre maintenant les inégalités sexuées, sociales et territoriales dans les quartiers de la politique de la ville et les territoires ruraux fragilisés, Rapport pour le Haut conseil à l'égalité entre les femmes et les hommes, La Documentation française, France, $286 \mathrm{p}$.

14. Ponthieux, S (2013) L'information statistique sexuée dans la statistique publique : état des lieux et pistes de réflexion, Rapport de recherche pour le Ministre des Droits des femmes, Insee.

15. Raibaud, Y (2014) «Une ville faite pour les garçons », Le Journal $d u C N R S$, en ligne.

16. Riddoch, C-J, Boandersen, L, Wedderkopp, N et al. (2004) « Physical activity levels and patterns of 9- and 15-yr-old European children », Medicine and Science in Sports and Exercise 36: 86-92.

17. Brougère, G (1999) « Les expériences ludiques des filles et des garçons ", In: Lemel, Y, Roudet, B (eds.), Filles et garçons jusqu'à l'adolescence, Socialisations différentielles, Paris, L'Harmattan, pp 199-222.

18. Zaidman, C (2007) « Jeux de filles, jeux de garçons », Les cahiers du Cedref 15: 283-292.

19. Glevarec, H (2010) «Les trois âges de la culture de la chambre », Ethnologie française 40: 19-30.

20. Bouhia, R, Garrouste, M, Lebrère, A et al. (2011) «Être sans diplôme aujourd'hui en France : quelles caractéristiques, quel parcours et quel destin?», Économie et Statistique 443: 93-105.

21. Campbell, M-K, Donner, A (1989) « Classification efficiency of multinomial logistic regression relative to ordinal logistic regression », Journal of the American Statistical Association 84: $587-591$.

22. Bourbonnais, R (2015) Économétrie, $9^{\mathrm{e}}$ édition, cours et exercices corrigés, Dunod, $392 \mathrm{p}$.

23. OCDE (2013) Regards sur l'éducation 2013: Les indicateurs de l'OCDE, Éditions OCDE, $452 \mathrm{p}$.

24. Sherwin, H, Chatterjee, K, Jain, J (2014) «An exploration of the importance of social influence in the decision to start bicycling in England», Transportation Research Part A 68: 2-45

25. Carver, A, Salmon, J, Campbell, K et al. (2005) « How Do Perceptions of Local Neighborhood Relate to Adolescents' Walking and Cycling?», American Journal of Health Promotion 20: 139-147.

26. Granié, M-A (2008) «Socialisation différenciée au risque : influence de l'identité sexuée sur le rapport au risque et à la règle», In : Granié, M-A (eds.), Genre, Risques, Éducation, Socialisation (GENRES). La psychologie du développement au service de la compréhension de la différence des sexes dans l'accidentologie routière, Rapport final sur subvention PREDIT/DSCR, Salon de Provence, Inrets, pp 13-63.

27. Granié, M-A (2011) « Différences de sexe et rôle de l'internalisation des règles sur la propension des enfants à prendre des risques à vélo », Recherche transports sécurité 27: 34-41.

28. Aboucaya, G (2014) Rapport d'évaluation. Taux de respect des sas vélo à Nantes, Cerema, Direction Territoriale Ouest, $85 \mathrm{p}$.

29. Razemon, Olivier (2014) «Les 10 raisons pour lesquelles les cyclistes commettent des infractions au code de la route ", Le Monde, http://transports.blog.lemonde.fr/2014/11/24/les-10raisons-pour-lesquelles-les-cyclistes-commettent-des-infractionsau-code-de-la-route/

30. Tilahun, N-Y, Levinson, D-M, Krizek, K-J (2007) « Trails, Lanes, or Traffic: Valuing Bicycle Facilities with an Adaptive Stated Preference Survey ", Transportation Research A: 287-301.

31. Dill, J, Gliebe, J (2008) «Understanding and Measuring Bicycling Behavior : A Focus on Travel Time and Route Choice », Bicycling 29: 1-70.

32. Garrard, J, Rose, G, Lo, S-K (2008) « Promoting Transportation Cycling for Women : The Role of Bicycle Infrastructure », Preventive Medicine 46: 55-59.

33. Zaffran, J (2016) «Bouger pour grandir. Défection et mobilité des adolescents », Annales de la recherche urbaine 111: 68-77.

34. Gilow, M (2015) «Déplacements des femmes et sentiment d'insécurité à Bruxelles : perceptions et stratégies », Brussels Studies 87: 1-11.

35. MTI Conseil et TNS Sofres (2013) Le vélo et les Français en 2012, $28 \mathrm{p}$.

36. Tremblay, M-S (2010) Condition physique des enfants et des jeunes au Canada : résultats de l'Enquête canadienne sur les mesures de la santé de 2007-2009, Rapport sur la santé 21: $1-16$.

37. Eschwege, E, Charles, M-A, Basdevant, A (2012) Enquête épidémiologique nationale sur le surpoids et l'obésité, Rapport Institut Roche de l'obésité, Inserm, Kantar-Health, 60 p.

38. Elliott, M-A, Baughan, C-J (2004) «Developing a self-report method for investigating adolescent road user behavior, Traffic Psychology and Behaviour», Transportation Research 7: 373-393.

39. Sullman M-J-, M, Mann, H-N (2009) «The road user behaviour of New Zealand adolescents. Traffic Psychology and Behaviour», Transportation Research 12: 494-502.

40. Amoros, E, Blaizot, S, Papon, F. et al. (2012) Rapport Accidentalité à Vélo et Exposition au risque (AVER), Risque de traumatismes routiers selon quatre types d'usagers, Rapport final sur la Convention DSCR, $172 \mathrm{p}$. 
41. Carver, A, Timperio, A, Hesketh, K et al. (2012) « How does perceived risk mediate associations between perceived safety and parental restriction of adolescents' physical activity in their neighborhood ? », International Journal of Behavioral Nutrition and Physical Activity 9: 1-57.

42. Rivière, C (2014) Ce que tous les parents disent? Approche compréhensive de l'encadrement parental des pratiques urbaines des enfants en contexte de mixité sociale (Paris-Milan), Thèse IEP de Paris, $470 \mathrm{p}$.

43. Mardon, A (2010) « Pour une analyse de la transition entre enfance et adolescence », Agora débats/jeunesses 54: $13-26$.

44. Adam, T (2013) Gynécologie du sport. Risques et bénéfices de l'activité physique chez la femme, Springer Science \& Business Media, $552 \mathrm{p}$.

45. O'Followell, L (1900) Bicyclette et organes génitaux, Paris, Baillière, $184 \mathrm{p}$.

46. Demeny, G (1903) Mécanisme et éducation des mouvements, Paris, Félix Alcan

47. Clair, I (2008) Les jeunes et l'amour dans les cités, Paris, Armand Colin.
48. Daude, H (2011) L'infirmière scolaire face à la demande de soins d'adolescents : Une approche spécifique?

49. Allaz, E (2014) Enquête sur la représentation et l'utilisation du vélo chez les adolescents. Étude d'efficacité du projet de promotion du vélo DÉFI VÉLO dans le canton de Vaud, Mémoire sous la direction de Marco Astolfi, Université de Lausanne, 134 p.

50. Kelly, P, Matthews, A, Foster, C (2012) Young and physically active : a blueprint for making physical activity appealing to youth, Rapport de recherche pour la World Health Organization, Regional Office for Europe Department of Public Health, University of Oxford, United Kingdom

51. Tibballs, S (2011) Changing the Game, for Girls, Rapport de recherche pour la Women's Sport and Fitness Foundation, Royaume-Uni.

52. Fraysse, M, Mennesson, C (2009) « Masculinités hégémoniques et féminités : les modèles de genre dans une revue de VTT », Sciences sociales et sport 2: 25-53.

53. Deschamps, E (2013) « Le vélo, un coup de pouce pour les femmes immigrées à Strasbourg », La Croix, http://www.lacroix.com/Solidarite/En-France/Le-velo-un-coup-de-pouce-pourles-femmes-immigrees-a-Strasbourg-2013-10-21-1047704. 